\title{
Short-term high glucose exposure impairs insulin signaling in endothelial cells
}

Valeria De Nigris ${ }^{1 *}$, Gemma Pujadas ${ }^{1}$, Lucia La Sala$^{1}$, Roberto Testa $^{2}$, Stefano Genovese ${ }^{3}$ and Antonio Ceriello ${ }^{{ }^{*}}$

\begin{abstract}
Background: Hyperglycemia is the hallmark of diabetes and its cardiovascular complications. Insulin plays an important role in the regulation of vascular homeostasis and maintenance of endothelial function. Insulin signaling occurs after binding to the insulin receptor, causing activation of two separate and parallel pathways: PI3K/AKT/eNOS and Ras/Raf/MAPK pathways. AKT phosphorylates eNOS at Ser1177, resulting in increased nitric oxide production and vasodilation. The MAPK pathway results in endothelin-1 production and vasoconstriction and mitogenic effects.
\end{abstract}

Methods: We studied the effects of physiological insulin treatment in human umbilical vein endothelial cells (HUVECS) on the two pathways under high glucose conditions, which mimic the in vivo condition of hyperglycemia. HUVECs were incubated with insulin at different physiological concentrations (from $10^{-10}$ to $10^{-8} \mathrm{M}$ ) for 30 min after $24 \mathrm{~h}$ of exposition to normal ( $5 \mathrm{mmol} / \mathrm{L}$, NG) or high glucose $(25 \mathrm{mmol} / \mathrm{L}, \mathrm{HG}$ ). Phosphorylated forms of AKT, eNOS, ERK1/2, p38, JNK and insulin receptor- $\beta$ subunit (IR $\beta$ ) were evaluated.

Results: In normal glucose, the active phosphorylated forms of AKT, eNOS, ERK1/2, p38 and JNK were increased in insulin treated cells, in a dose-dependent manner. In high glucose, insulin was not able to activate the PI3K/AKT/ eNOS pathway, with the phosphorylated form of eNOS reduced with respect to the control. However, insulin was able to induce the up-regulation of phospho-ERK1/2,-p38 and -JNK. Moreover, we found reduced levels of IR $\beta$ phosphorylated form in high glucose as compared to the control. Insulin was able to increase phospho-IR $\beta$ in normal glucose but not in high glucose, in which the total protein levels remained reduced.

Conclusions: Exposure to short-term high glucose negatively affects insulin signaling even when physiological insulin concentrations are added. The impairment of the PI3K/AKT/eNOS pathway after physiological insulin treatment could contribute to detrimental effects on cardiovascular homeostasis under high glucose conditions, and might shift toward the activation of certain mitogenic effectors, such as ERK1/2, p38 and JNK, the only ones that respond to physiological insulin treatment in high glucose.

Keywords: Physiological insulin, High glucose, eNOS, Endothelium, HUVECs

\section{Background}

The pathogenesis of cardiovascular complications in diabetes has been largely described and begins with endothelial dysfunction [1]. The endothelium plays a crucial role in maintaining cardiovascular homeostasis under physiological conditions by releasing a number of

\footnotetext{
*Correspondence: vnigris@clinic.ub.es; aceriell@clinic.ub.es

1 Insititut d'Investigacions Biomèdiques August Pi i Sunyer (IDIBAPS)

and Centro de Investigación Biomédica en Red de Diabetes y

Enfermedades Metabólicas Asociadas (CIBERDEM), Hospital Clinic,

C/Rosselló, 149-153, 08036 Barcelona, Spain

Full list of author information is available at the end of the article
}

vasoactive substances, vasodilators and vasoconstrictors, in balance. They act together to regulate vascular tone, antiproliferation and antiaggregation, and, in addition, to limit increases in blood pressure, to control tissue blood flow and inflammatory responses [2, 3]. The endothelium releases these vasoactive substances in response to mechanical stimuli, such as pressure and shear stress, as well as various hormonal stimuli, such as insulin.

Normal insulin signaling results in glucose uptake by skeletal muscle and fat, suppression of hepatic gluconeogenesis and vasodilation for increased eNOS enzymatic activity in the endothelium [4]. These effects are 
mediated by insulin binding to the insulin receptor (IR), a ligand-activated tyrosine kinase receptor. Binding and activation of IR results in tyrosine phosphorylation of insulin receptor substrates (IRS) and Shc, leading to the activation of two parallel pathways: the PI3K/AKT/eNOS pathway, also known as the "metabolic arm", and the Ras/ Raf/MAPK pathway, or the "mitogenic/pro-atherogenic arm". Phosphorylation of IRS-1 leads to the activation of the PI3K/AKT/eNOS pathway. AKT kinase phosphorylates eNOS at Ser1177 and activates it, leading to the production of nitric oxide (NO). On the other hand, phosphorylation of Shc leads to activation of the Ras/Raf/ MAPK pathway, resulting in increased endothelin-1 (ET1) expression and mitogenic effects $[5,6]$.

Insulin resistance is an important feature of diabetes, obesity, glucose intolerance and dyslipidemia, and it is also a prominent component of cardiovascular disorders, including hypertension, coronary artery disease, and atherosclerosis [7]. In the endothelium, a selective resistance to insulin is observed in the pathological state of hyperinsulinemia [8, 9], when the PI3K/AKT/eNOS pathway is altered, resulting in diminished eNOS activity, reduced NO generation and diminished insulin-mediated vasodilation; by contrast, the Ras/Raf/MAPK pathway is generally preserved, thus ET-1 production and mitogenic effects persist contributing to the vascular effects of insulin resistance [10]. Several studies have investigated the consequences of high insulin levels in vitro and in vivo. In vitro, in human coronary artery endothelial cells (HCAECs) cultured in the presence of $20 \mathrm{mmol} / \mathrm{L}$ glucose and treated with an insulin concentration of $50 \mathrm{nmol} / \mathrm{L}$ for $10 \mathrm{~min}$, a reduction in insulin-stimulated phosphorylation of eNOS at Ser1177 was observed [11]. In vivo, Kubota et al. [12] demonstrated that the expression levels of insulin-stimulated phosphorylations of AKT and eNOS were decreased by $70-80 \%$ in endothelial cells extracted from the abdominal aorta of 8-week high-fat diet-fed mice and ob/ob mice, treated with an insulin concentration of $100 \mathrm{nmol} / \mathrm{L}$ for $30 \mathrm{~min}$, indicating that insulin signaling was impaired in the endothelial cells of these obesity models. While several reports have investigated the role of hyperinsulinemia in the endothelium [11-13] and in the liver [14] and muscle [14, 15], studies in which insulin is used at physiological concentrations are lacking.

The present study aimed to investigate the role of insulin at physiological levels on vascular endothelium in the presence of high glucose concentrations. We hypothesized that the impairment of the insulin signaling may occur in endothelial cells exposed to high glucose, not only at high insulin levels but also at physiological insulin concentrations. In this way, we could help clarify the contribution of insulin to endothelial phenotypes altered by high glucose.

\section{Methods}

\section{Materials}

D-Glucose and insulin were purchased from SigmaAldrich (St. Louis, MO, USA).

\section{Cell culture}

HUVECs were purchased from Lonza and cultured in an endothelial cell growth medium-2 (EBM-2) (Lonza Bioresearch LBS, Basel, Switzerland) supplemented with hEGF, FBS, hFGF, heparin, hydrocortisone and GA-1000. It contained less than $10^{-12} \mathrm{M}$ insulin, which was considered to have no effect on our outcome [16]. Cells were harvested at subconfluence and seeded into six-well plates.

\section{Experimental design}

The effects of various concentrations of insulin were examined in HUVECs cultured under normal glucose $(5 \mathrm{mmol} / \mathrm{L}, \mathrm{NG})$ and high glucose conditions ( $25 \mathrm{mmol} / \mathrm{L}, \mathrm{HG}$ ) for $24 \mathrm{~h}$. In the last $30 \mathrm{~min}$, HUVECs were treated with increasing physiological concentrations of insulin: $10^{-10} \mathrm{M}=100 \mathrm{pmol} / \mathrm{L}, 10^{-9} \mathrm{M}=10 \mathrm{nmol} / \mathrm{L}$ and $10^{-8} \mathrm{M}=1 \mathrm{nmol} / \mathrm{L}$. After insulin treatment, cells were collected for protein extraction and Western Blot analysis.

\section{Protein extraction}

Cells were harvested and whole cell lysates were prepared using a RIPA buffer (Sigma-Aldrich, St. Louis, MO, USA) with the addition of a protease and phosphatase inhibitor cocktail. Protein content of the lysates was determined using the Bradford reagent (Sigma-Aldrich, St. Louis, MO, USA).

\section{Western blot analysis}

Protein lysates ( $25 \mathrm{ug}$ ) were resolved by SDS-polyacrylamide gel electrophoresis (PAGE-R Gold gels 4-20\%, purchased from Lonza) and transferred to a polyvinylidene fluoride (PVDF) membrane. After blocking with $5 \%$ non-fat dry milk (NFDM) in $20 \mathrm{mM}$ Tris- $\mathrm{HCl}$ (pH 7.5), $135 \mathrm{mM} \mathrm{NaCl}$ and $0.1 \%$ Tween-20, blots were incubated with monoclonal antibodies against human phospho-AKT (Ser473), phospho-eNOS (Ser1177), phospho-ERK1/2 (Thr202/Tyr204), phospho-p38 (Thr180/Tyr182), phospho-JNK (Thr183/Tyr185), AKT, eNOS, ERK1/2, p38 and JNK (Cell Signaling Technology, Beverly, MA, USA), IR $\beta$ (Santa Cruz Biotechnology, CA, USA) and phosphotyrosine (phospho-Tyr) (UBI, Upstate, Lake Placid, NY, USA) (1:1000). Human 
$\beta$-actin (1:1000) (Sigma-Aldrich, St. Louis, MO, USA) was used as a loaded control. Detection was performed using a secondary peroxidase-linked anti-mouse/rabbit antibody (1:3000) (GE Healthcare Europe GmbH, Barcelona, Spain) and an enhanced chemiluminescence system (Pierce Chemical Co, Rockford, IL, USA) according to the manufacturer's instructions. Proteins were revealed in a CCD camera (ImageQuantLAS4000, GE Healthcare, UK). Protein content quantification was performed using computer-assisted densitometry (http://www.imagej.nih. gov, ImageJ Software, NIH).

\section{Results}

Acute high glucose enhanced AKT phosphorylation and attenuated eNOS phosphorylation in HUVECs

PI3K/AKT/eNOS is one of the two parallel pathways activated buy insulin after binding to its receptor [5]. Particularly, AKT is an important signaling molecule that is involved in different endothelial functions, for example the regulation of angiogenesis, proliferation, vascular permeability, survival and cellular transformation as well [17]. Also AKT may phosphorylate eNOS to promote vasodilation with increased NO production in endothelial cells [5].

It has been shown that impaired PI3K/AKT signaling due to hyperglycemia may promote endothelial dysfunction in diabetes [18]. To this aim, we firstly investigated the effects of acute HG in AKT phosphorylation and in its effector eNOS in HUVECs. In high glucose, phosphorylation levels of Ser473AKT increased, while phosphorylation levels of Ser1177eNOS reduced versus the control. The total amount of AKT and eNOS protein levels remained unchanged (Fig. 1). These results suggest that acute HG impairs AKT/eNOS signal transduction.

\section{Time course of insulin-mediated eNOS phosphorylation at Ser1177 in HUVECs}

In order to establish the optimal amount of time for insulin incubation, confluent HUVECs were treated with $100 \mathrm{pmol} / \mathrm{L}$ insulin at three different time points: 5, 10 and $30 \mathrm{~min}$. The insulin concentration used was $100 \mathrm{pmol} / \mathrm{L}$ insulin because it corresponds to physiological plasma insulin levels in humans, so this amount may be considered a good in vitro representation of in vivo insulin activity [16]. After insulin treatment, cells were collected and eNOS phosphorylation in Ser1177 levels were analyzed by Western Blot. We observed gradually increasing levels of Ser1177eNOS between 5 and $30 \mathrm{~min}$, reaching statistical significance at $30 \mathrm{~min}$ (Fig. 2).

\section{Physiological insulin administration enhanced AKT and eNOS phosphorylation in NG in a} concentration-dependent manner, but had no effects in HG in HUVECs

To investigate the alterations on the PI3K/AKT/ eNOS-dependent insulin signaling pathway under HG
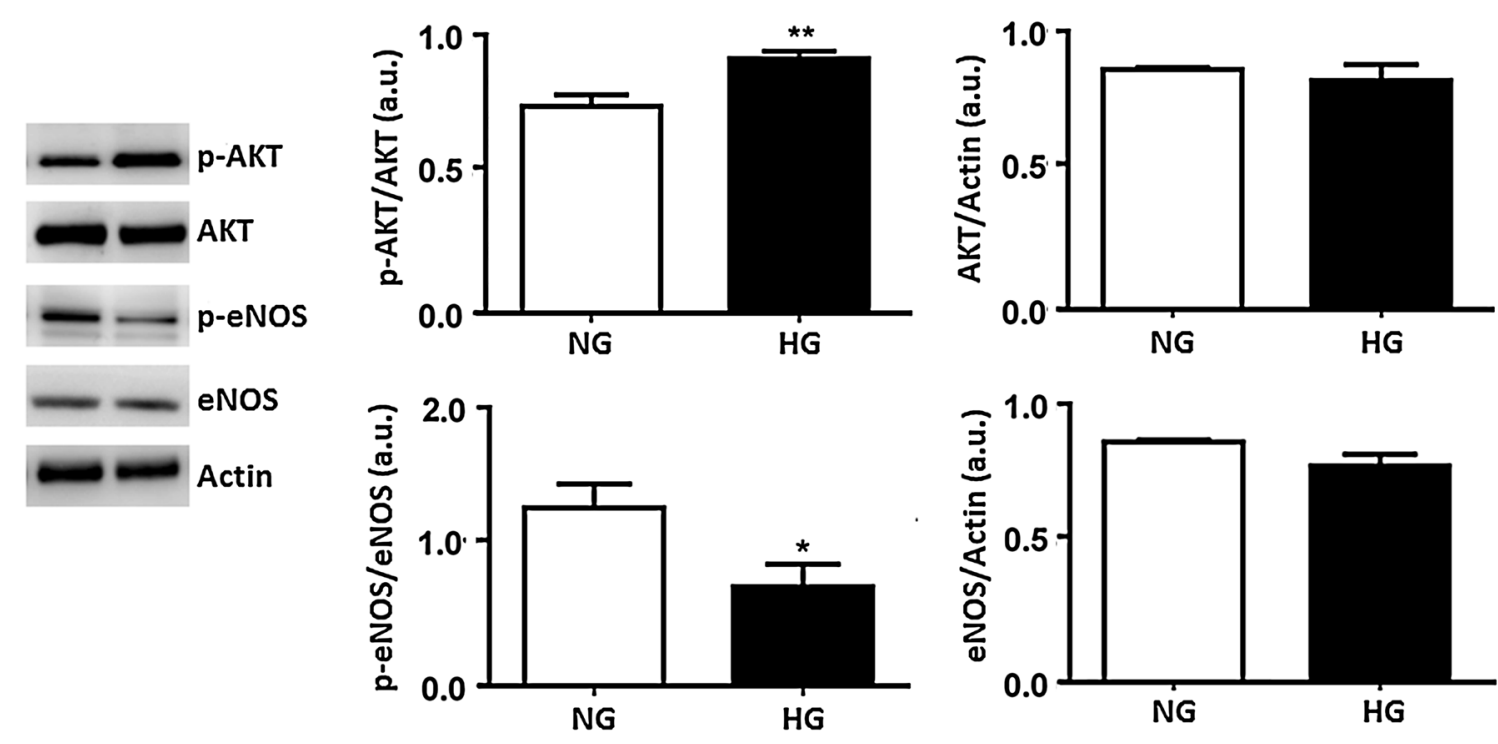

Fig. 1 Administration of high glucose for $24 \mathrm{~h}$ enhanced phosphor-Ser473AKT and attenuated phospho-Ser1177eNOS in HUVECs. Whole cell lysates were prepared from confluent HUVECs exposed to $5 \mathrm{mmol} / \mathrm{L}$ (NG) or $25 \mathrm{mmol} / \mathrm{L}$ (HG) glucose for $24 \mathrm{~h}$. AKT and eNOS phosphorylations were assayed for Western Blot analysis. The panels show representative images of different independent experiments. Densitometric values were normalized to total amounts of AKT and eNOS, respectively. ${ }^{*} \mathrm{P}<0.05$, ${ }^{* *} \mathrm{P}<0.01 \mathrm{HG}$ vs. NG. Bars represent mean \pm SEM for five independent experiments 


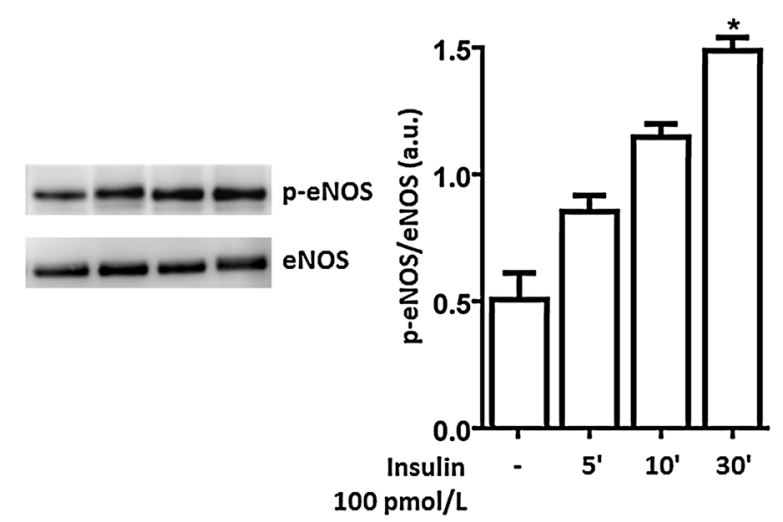

Fig. 2 Insulin $100 \mathrm{pmol} / \mathrm{L}$ slightly increased phospho-Ser1177eNOS at 5 and $10 \mathrm{~min}$, with its major effect occurring at $30 \mathrm{~min}$. HUVECs were treated with $100 \mathrm{pmol} / \mathrm{L}$ insulin at different time points: 5, 10 and $30 \mathrm{~min}$. Whole cell lysates were prepared for Western Blot analysis. The panels show representative images of different independent experiments. Densitometric values were normalized to total eNOS. Bars represent mean \pm SEM for five independent experiments. ${ }^{*} \mathrm{P}<0.05 \mathrm{NG}$ with insulin $100 \mathrm{pmol} / \mathrm{L}$ vs. NG w/o insulin treatment

conditions, activation of AKT and eNOS was determined by immunoblotting. Insulin administered at different physiological concentrations under NG conditions caused a concentration-dependent increase in phosphoSer473AKT, which was statistically significant at the concentration of $10^{-9}$ and $10^{-8} \mathrm{M}$ (Fig. 3). Contrary to what happened under NG, insulin physiological treatment under HG conditions did not cause the same effect.

To determine whether such a difference in endothelial AKT activity in response to insulin concentrations under the two conditions of NG and HG also had downstream consequences, we next evaluated the phosphorylation of eNOS. The different physiological insulin concentrations added at NG induced an increase in phosphoSer1177eNOS that nicely correlated to a dose between $10^{-10}$ and $10^{-9} \mathrm{M}$, but was attenuated at $10^{-8} \mathrm{M}$. This could indicate a desensitization of the metabolic insulin signaling pathway. However, as the same insulin concentration continued to increase phosphorylated AKT levels, this indicated that insulin preserved some ability to signal to the metabolic arm.

Interestingly, physiological insulin added to HUVECs acutely treated under high glucose conditions did not follow the same eNOS phosphorylation profile that we observed in NG, indicating that insulin could not prevent high glucose-dependent inhibition of eNOS activation.

\section{Acute high glucose had no effects on ERK1/2, JNK and p38 phosphorylation levels in HUVECs, and physiological insulin treatment increased them under NG and HG conditions}

To investigate the alterations in the Ras/Raf/MAPKdependent insulin signaling pathway in HG, activation of ERK1/2, p38 and JNK was determined. In NG, HUVECs

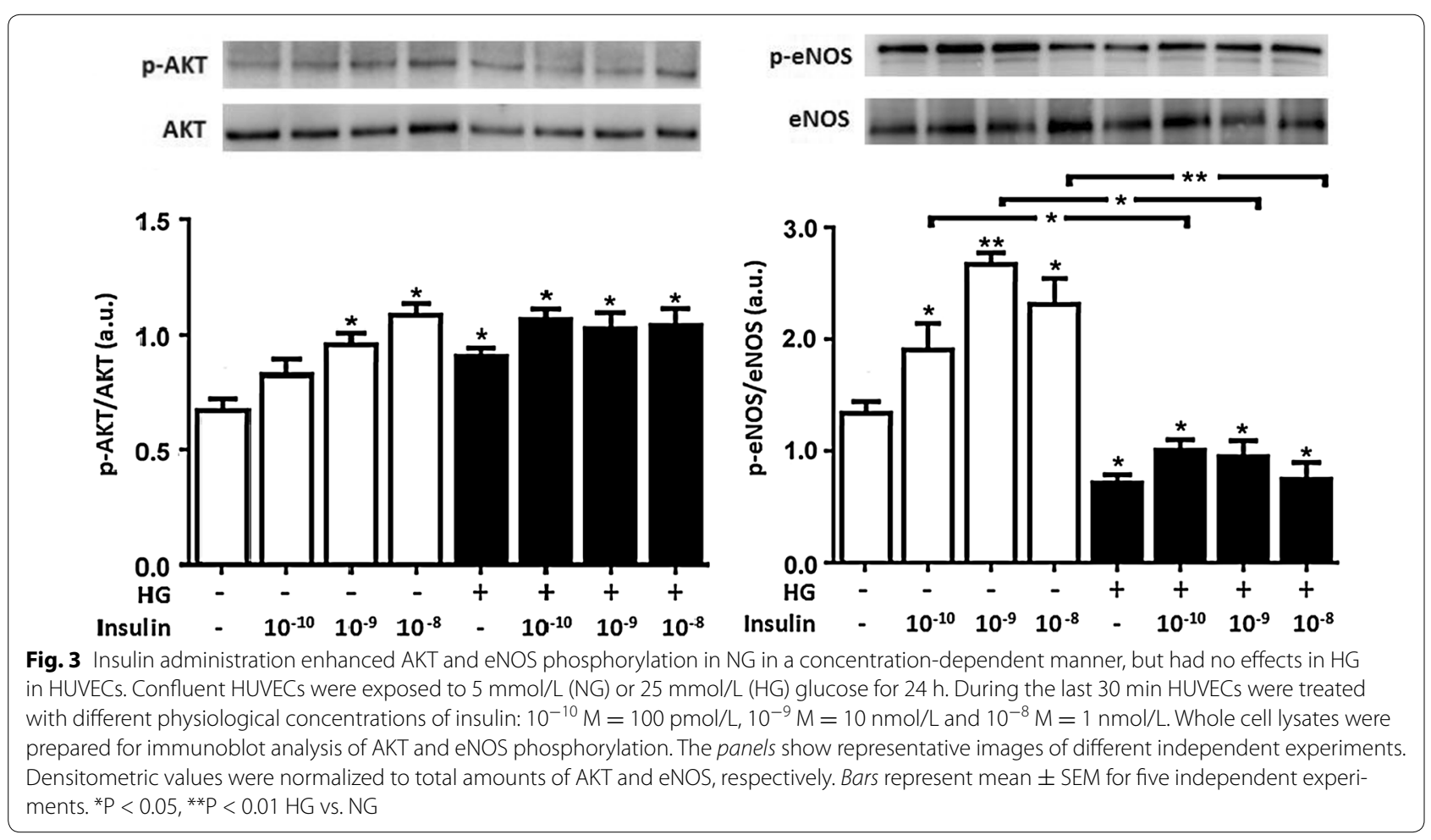


responded to insulin stimulation with an increase in ERK1/2 phosphorylation in a concentration-dependent manner, being statistically significant for insulin doses $10^{-9}$ and $10^{-8} \mathrm{M}$. We obtained the same results for phospho-p38, which was statistically significant at insulin doses of $10^{-8} \mathrm{M}$, and phospho-JNK, with a significant increase at the insulin concentrations of $10^{-9}$ and $10^{-8} \mathrm{M}$ $(\mathrm{p}<0.05)$. Conversely to AKT and eNOS phosphorylation, $24 \mathrm{~h}$ of HG treatment did not affect the phosphorylated forms of ERK1/2, p38 or JNK. Intriguingly, insulin physiological treatment in HG maintained an increase of these forms in a concentration-dependent manner, and this increase was significant (1) at all insulin doses tested for phospho-ERK1/2, (2) at the concentration of $10^{-8} \mathrm{M}$ for phospho-p38 and (3) at the concentrations of $10^{-9}$ and $10^{-8} \mathrm{M}$ for phospho-JNK (Fig. 4).

Acute high glucose attenuated IR $\beta$ expression in HUVECs. Insulin administration had no effects under NG and HG in terms of total amount but increased its phosphorylated form

We hypothesized that exposure of HUVECs to HG could affect the early steps of insulin signaling. To test this, IR $\beta$ protein expression levels were examined. Acute exposure of HUVECs to HG induced a significant decrease in IR $\beta$ tyrosine phosphorylation accompanied by a reduction in IR $\beta$ total protein versus the control (Fig. 5).

Insulin treatment under NG conditions enhanced in a dose-dependent manner IR $\beta$ tyrosine phosphorylation at all tested physiological insulin concentrations, but it had no effects under HG in HUVECs, although a statistically insignificant increase was observed. Insulin stimulation in NG had no effects on IR $\beta$ total expression compared with the untreated control. The decrease in IR $\beta$ total expression observed under HG conditions was not counteracted by any tested dose of insulin (Fig. 5).

\section{Discussion}

In the present study, we show how HUVECs exposed to $24 \mathrm{~h}$ of high glucose lose the capacity to respond normally at physiological insulin concentrations. This phenomenon is only observed in the PI3K/AKT/eNOS pathway, while under the same conditions the Ras/Raf/ MAPK pathway responds to insulin treatment with the up-regulation of phosphorylated forms of ERK1/2, p38 and JNK. This is consistent with the hypothesis that an imbalance occurs in the endothelial insulin signaling between pro- and anti-atherogenic arms with insulin preserving its ability to signal through the mitogenic arm, previously shown not to be affected under conditions of high insulin levels [19-21]. In a novel manner, we show that stimulation of endothelial insulin signaling with physiological insulin concentrations in HUVECs pre-exposed to $24 \mathrm{~h}$ high glucose: (1) on the one hand, is not sufficient to activate the PI3K/AKT/eNOS pathway, as has occurred in other studies involving lower insulin dose incubations [11, 22]; (2) and on the other, it does not affect the MAPK-mediated insulin pathway, which not only is maintained but also is exacerbated, and this occurs at very low insulin concentrations.

An altered PI3K/AKT/eNOS pathway is a common feature observed in cellular models exhibiting impaired endothelial function [5]. eNOS phosphorylation at Ser1177 is necessary for its maximal activation and, consequently, for optimal NO production [23]. In the present study, we show that in HUVECs expression of

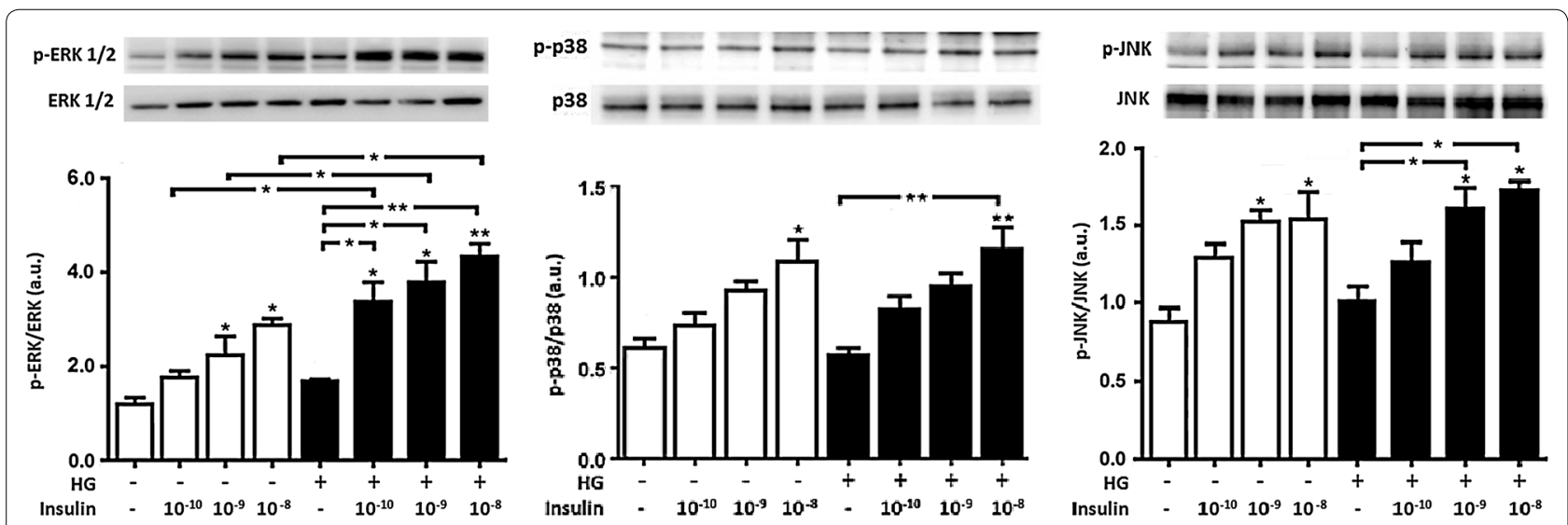

Fig. 4 Administration of high glucose for $24 \mathrm{~h}$ had no effects on ERK1/2, p38 and JNK phosphorylations in HUVECs; however, insulin administration increased them under NG and HG conditions. Confluent HUVECs were exposed to $5 \mathrm{mmol} / \mathrm{L}$ (NG) or $25 \mathrm{mmol} / \mathrm{L}$ (HG) of glucose for $24 \mathrm{~h}$. During the last 30 min, HUVECs were treated with different physiological concentrations of insulin, and whole cell lysates were prepared for Western Blot analysis. The panels show representative images of different independent experiments. Densitometric values were normalized to total amounts of ERK1/2, p38 and JNK, respectively. Bars represent mean \pm SEM for five independent experiments. ${ }^{*} \mathrm{P}<0.05,{ }^{*} \mathrm{P}<0.01 \mathrm{HG}$ vs. NG 

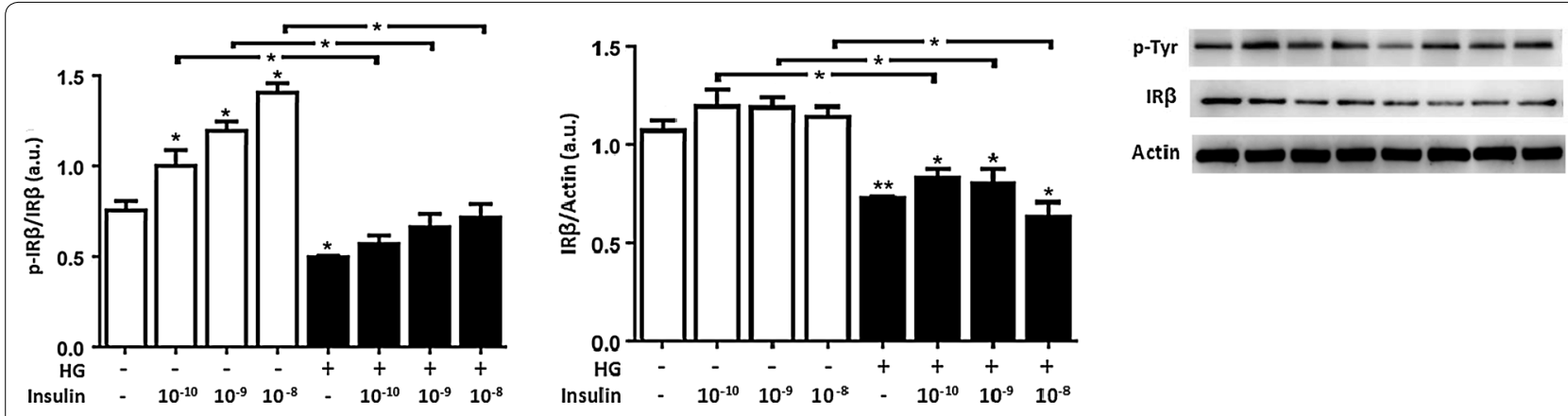

Fig. 5 Administration of high glucose for $24 \mathrm{~h}$ attenuated IR $\beta$ total expression and its phosphorylated form in HUVECs. Physiological insulin increased IR $\beta$ tyrosine phosphorylation only for the condition of NG. Previously exposed to $24 \mathrm{~h}$ of $5 \mathrm{mmol} / \mathrm{L}$ ( $\mathrm{NG}$ ) or $25 \mathrm{mmol} / \mathrm{L}$ ( $\mathrm{HG}$ ) of glucose, cells were stimulated with different insulin concentrations for $30 \mathrm{~min}$. Whole cell lysates were prepared for immunoblot analysis of the phosphorylated form of IR $\beta$ and the total amount of IR $\beta$ protein. The panels show representative images of different independent experiments. Densitometric values were normalized to total amounts of IR $\beta$ and actin, as indicated. Bars represent mean \pm SEM for five independent experiments. ${ }^{*} \mathrm{P}<0.05$, **P $<0.01 \mathrm{HG}$ vs. NG

phospho-eNOS is decreased after $24 \mathrm{~h}$ of high glucose and that physiological insulin treatment under this condition cannot restore this activation.

PI3K and AKT are downstream effectors of insulin signaling and are also involved in other pathways implicated in proliferation and survival [24], apoptosis, angiogenesis and cellular transformation [17]. In our study, we observe that, when HUVECs are exposed to $24 \mathrm{~h}$ of high glucose, eNOS is not activated, while phospho-AKT is increased. These results suggest that under high glucose conditions AKT is probably directed to positively regulate proliferation and endothelial cell survival.

Attenuated insulin signaling has been proposed as resulting from alterations in both the IR and postreceptor signaling [25]. The IR is a heterodimeric protein complex, with an intracellular $\beta$-subunit (IR $\beta$ ) and an extracellular $\alpha$-subunit (IR $\alpha)$. Insulin binds to the IR through IR $\alpha$, while the $\beta$ subunit contains the intracellular tyrosin-kinase domain that initiates the signal cascade through the cytoplasm and the nucleus.

In the present study, we show that exposure to high glucose not only affects downstream IR signaling events, but also the total amount of intracellular $\beta$ subunit. Under high glucose conditions, insulin is not able to phosphorylate the IR $\beta$ subunit, while it occurs under normal glucose conditions. Furthermore, IR $\beta$ total expression, which is decreased by high glucose, is unable to be restored through insulin treatment back to normal protein levels.

The decrease of IR $\beta$ total expression levels observed under high glucose potentiates the hypothesis that elevated AKT phosphorylation in this condition has consequences not only for eNOS activation, which directly depends on insulin signaling, but also for other effects governed by other pathways [17].

\section{Conclusions}

Our study suggests that insulin at physiological concentrations is not preventive but may even contribute to the vascular impairment of endothelial phenotype previously damaged by high glucose, due to an increased pro-atherogenic pathway observed by an up-regulation of ERK1/2, p38 and JNK phosphorylation.

\section{Abbreviations}

PI3K: phosphatidylinositol-4,5-bisphosphate 3-kinase; AKT: protein kinase B; eNOS: endothelial nitric oxide synthase; NO: nitric oxide; MAPK: mitogenactivated protein kinase; ERK1/2: extracellular signal-regulated kinase 1/2; JNK: jun-amino(N)-terminal kinase; ET-1: endothelin-1; HUVECs: human vein endothelial cells; NG: normal glucose; HG: high glucose; Ins: insulin; IR- $\alpha / \beta$ : insulin receptor-alpha/beta subunit; IRS-1/2: insulin receptor substrates-1/2; NG: normal glucose; HCAECs: human coronary artery endothelial cells.

\section{Authors' contributions}

VDN, contributed to: researched data, discussion, wrote manuscript, reviewed/ edited manuscript. GP, contributed to: researched data, discussion. LLS, contributed to: researched data, discussion. RT, contributed to: researched data, discussion. SG, contributed to: discussion, reviewed/edited manuscript. AC, contributed to: researched data, discussion, wrote manuscript, reviewed/ edited manuscript. All authors read and approved the final manuscript.

\section{Author details}

${ }^{1}$ Insititut d'Investigacions Biomèdiques August Pi i Sunyer (IDIBAPS) and Centro de Investigación Biomédica en Red de Diabetes y Enfermedades Metabólicas Asociadas (CIBERDEM), Hospital Clinic, C/Rosselló, 149-153, 08036 Barcelona, Spain. ${ }^{2}$ Experimental Models in Clinical Pathology, INRCA-IRCCS National Institute, Ancona, Italy. ${ }^{3}$ Department of Cardiovascular and Metabolic Diseases, IRCCS Gruppo Multimedica, Sesto San Giovanni, MI, Italy.

\section{Acknowledgements}

The authors wish to thank Kimberly Katte of CIBERDEM for assistance in preparing the manuscript.

Compliance with ethical guidelines

\section{Competing interests}

The authors declare that they have no competing interests.

Received: 31 March 2015 Accepted: 12 August 2015

Published online: 22 August 2015 


\section{References}

1. Brownlee M. Biochemistry and molecular cell biology of diabetic complications. Nature. 2001;414:813-20.

2. Tabit CE, Chung WB, Hamburg NM, Vita JA. Endothelial dysfunction in diabetes mellitus: molecular mechanisms and clinical implications. Rev Endocr Metab Disord. 2010;11:61-74.

3. Van den Oever IAM, Raterman HG, Nurmohamed MT, Simsek S. Endothelial dysfunction, inflammation, and apoptosis in diabetes mellitus. Mediat Inflamm. 2010;2010:792393-408.

4. Kim J, Montagnani M, Koh KK, Quon MJ. Reciprocal relationships between insulin resistance and endothelial dysfunction: molecular and pathophysiological mechanisms. Circulation. 2006;113:1888-904.

5. Nystrom FH, Quon M. Insulin signalling: metabolic pathways and mechanisms for specificity. Cell Signal. 1999;11:563-74.

6. Saltiel A, Kahn C. Insulin signalling and the regulation of glucose and lipid metabolism. Nature. 2001;414:799-806.

7. Cersosimo E, DeFronzo RA. Insulin resistance and endothelial dysfunction: the road map to cardiovascular diseases. Diabetes Metab Res Rev. 2006;22:423-36.

8. Muniyappa R, Sowers JR. Role of insulin resistance in endothelial dysfunction. Rev Endocr Metab Disord. 2013;14:5-12.

9. Muniyappa R, Montagnani M, Koh KK, Quon MJ. Cardiovascular actions of insulin. Endocr Rev. 2007;28:463-91.

10. Huang PL. eNOS, metabolic syndrome and cardiovascular disease. Trends Endocrinol Metab. 2009;20:295-302.

11. Federici M. Insulin-dependent activation of endothelial nitric oxide synthase is impaired by O-linked glycosylation modification of signaling proteins in human coronary endothelial cells. Circulation. 2002;106:466-72.

12. Kubota T, et al. Impaired insulin signaling in endothelial cells reduces insulin-induced glucose uptake by skeletal muscle. Cell Metab. 2011;13:294-307.

13. Taguchi K, et al. Tonic inhibition by G protein-coupled receptor kinase 2 of Akt/endothelial nitric-oxide synthase signaling in human vascular endothelial cells under conditions of hyperglycemia with high insulin levels. J Pharmacol Exp Ther. 2014;349:199-208.

14. Saad MJ, et al. Regulation of insulin receptor substrate-1 in liver and muscle of animal models of insulin resistance. J Clin Invest. 1992;90:1839-49.
15. Bertacca A, et al. Continually high insulin levels impair Akt phosphorylation and glucose transport in human myoblasts. Metabolism. 2005;54:1687-93.

16. Matsui-hirai $\mathrm{H}$, et al. Dose-dependent modulatory effects of insulin on glucose-induced endothelial senescence in vitro and in vivo: a relationship between telomeres and nitric oxide. 2011;337:591-9.

17. Lee $\mathrm{C}-\mathrm{H}$, et al. High glucose induces human endothelial dysfunction through an Axl-dependent mechanism. Cardiovasc Diabetol. 2014;13:53

18. Varma S, et al. Hyperglycemia alters PI3k and Akt signaling and leads to endothelial cell proliferative dysfunction. Am J Physiol Heart Circ Physiol. 2005;289:H1744-51.

19. Montagnani M, et al. Inhibition of phosphatidylinositol 3-kinase enhances mitogenic actions of insulin in endothelial cells. J Biol Chem. 2002;277:1794-9.

20. Madonna R, Massaro M, Pandolfi A, Consoli A, De CR. The prominent role of p38 mitogen-activated protein kinase in insulin-mediated enhancement of VCAM-1 expression in endothelial cells. Int J Immunopathol Pharmacol. 2007;20:539-55.

21. Madonna R, De Caterina R. Prolonged exposure to high insulin impairs the endothelial PI3-kinase/Akt/nitric oxide signalling. Thromb Haemost. 2009;101:345-50. doi:10.1160/TH08-07-0484.

22. Pirola $L$, et al. Phosphoinositide 3-kinase-mediated reduction of insulin receptor substrate-1/2 protein expression via different mechanisms contributes to the insulin-induced desensitization of its signaling pathways in L6 muscle cells. J Biol Chem. 2003;278:15641-51.

23. Dou J, et al. Osteocalcin attenuates high fat diet-induced impairment of endothelium-dependent relaxation through Akt/eNOS-dependent pathway. Cardiovasc Diabetol. 2014;13:74.

24. Caporali A, et al. Neurotrophin p75 receptor (p75NTR) promotes endothelial cell apoptosis and inhibits angiogenesis: implications for diabetes-induced impaired neovascularization in ischemic limb muscles. Circ Res. 2008;103:e15-26.

25. Heydrick SJ, et al. Defect in skeletal muscle phosphatidylinositol-3-kinase in obese insulin- resistant mice. J Clin Invest. 1993;91:1358-66.

\section{Submit your next manuscript to BioMed Central and take full advantage of:}

- Convenient online submission

- Thorough peer review

- No space constraints or color figure charges

- Immediate publication on acceptance

- Inclusion in PubMed, CAS, Scopus and Google Scholar

- Research which is freely available for redistribution

Submit your manuscript at

www.biomedcentral.com/submit

C BioMed Central 